\title{
Michael McCarthy (dir.), Cambridge Word Routes: lexique thématique de l'anglais courant
}

Cambridge : Cambridge University Press, 1994

Lesley Graham

\section{OpenEdition}

\section{Journals}

Édition électronique

URL : http://journals.openedition.org/asp/4119

DOI : 10.4000/asp.4119

ISSN : 2108-6354

\section{Éditeur}

Groupe d'étude et de recherche en anglais de spécialité

Édition imprimée

Date de publication : 1 décembre 1994

Pagination : 262-263

ISSN : 1246-8185

\section{Référence électronique}

Lesley Graham, "Michael McCarthy (dir.), Cambridge Word Routes : lexique thématique de l'anglais courant », ASp [En ligne], 5-6 | 1994, mis en ligne le 20 décembre 2013, consulté le 22 septembre 2020. URL : http://journals.openedition.org/asp/4119; DOI : https://doi.org/10.4000/asp.4119

Ce document a été généré automatiquement le 22 septembre 2020.

Tous droits réservés 


\section{Michael McCarthy (dir.), Cambridge Word Routes : lexique thématique de l'anglais courant}

Cambridge : Cambridge University Press, 1994

Lesley Graham

\section{RÉFÉRENCE}

Michael McCarthy (dir.), Elizabeth Walter (dir. adj.). 1994. Cambridge Word Routes : lexique thématique de l'anglais courant. Cambridge : Cambridge University Press, 467 p. ISBN 0-521-42583-2. 
1 L'originalité de ce recueil lexical réside dans le fait qu'il regroupe les mots et expressions de sens voisins sous une même rubrique tout en fournissant des équivalents français et des explications en français.

C'est cet élément bilingue qui fait la différence avec, par exemple, le Longman Language Activator: The World's First Production Dictionary (1993).

3 Ainsi Cambridge Word Routes peut s'utiliser comme un dictionnaire. Il permet en plus de saisir la nuance qui sépare des mots proches, sans avoir à chercher ces mots à différents endroits. Il sert également de dictionnaire de synonymes pour l'utilisateur qui veut s'exprimer sur un sujet spécifique (médecine, bateaux, récipients,

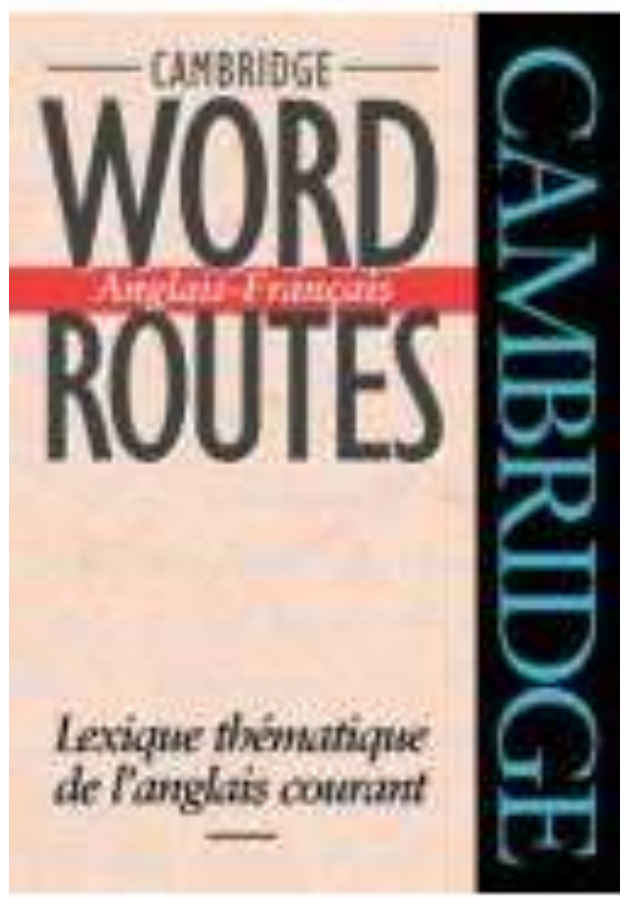
mouvement) et souhaite avoir une vue générale de ce champ sémantique avant de se lancer dans la production. Ce regroupement de mots par catégories se prête également à d'heureuses découvertes accidentelles, même si c'est en vain que l'on cherchera le mot « serendipity » dans Word Routes.

4 Bien que le lexique se limite délibérément à la langue de tous les jours, l'angliciste de spécialité y trouvera matière à consulter, voire à didactiser. De même, s'il est à l'origine conçu pour un public francophone, l'utilisateur anglophone de Cambridge Word Routes pourra se servir de cet outil, mais sera souvent frustré par l'absence d'informations d'ordre grammatical sur les mots et expressions français.

5 La typographie est claire et attrayante. Les commentaires sur les expressions idiomatiques et les proverbes, et surtout les notes sur l'emploi des mots sont très utiles: les nuances de sens s'en trouvent élucidées de manière précise et visible. Les entrées signalent systématiquement les différences entre les utilisations britannique et américaine. Des illustrations ponctuelles, bien que quelque peu ternes, sont les bienvenues. De temps en temps un encadré vient rajouter des informations d'ordre culturel à propos, par exemple, des systèmes politiques de la Grande-Bretagne et des États-Unis; les soins médicaux en Grande-Bretagne (mais pas aux États-Unis). Nous n'avons relevé que peu d'erreurs ; «network television » n'est pas la télévision par câble.

Dans l'introduction on lit que « les catégories de mots sont regroupées logiquement et de façon pratique ", les champs sémantiques voisins se trouvant à proximité les uns des autres. L'ordre attribué à ces différentes catégories reste, cependant, inexpliqué et l'on peut regretter l'absence d'un index de catégories des mots. L'utilisateur comprendra aisément que la catégorie 184 Personal Hygiene soit suivie de 185 Bathroom, mais le schéma conducteur sera moins évident lorsqu'immédiatement après la catégorie 226 Selfish il trouve 227 Politics and Government, et 152 Fruits après 151 Emotion. Il nous semble qu'un index montrant clairement l'organisation des différents ensembles de 
catégories aurait guidé l'utilisateur dans sa recherche, qu'il aurait fait fonction en quelque sorte de carte routière, complétant ainsi les indications des panneaux de signalisation que constituent les titres et les sous-titres de ces Word Routes.

7 Les deux index de mots, anglais et français, sont en revanche de la plus grande utilité, d'autant que la transcription phonétique est donnée, seulement, il est vrai, pour la partie anglaise de l'index : l'ouvrage est en effet explicitement destiné aux apprenants francophones. Le fait qu'il existe un « directeur de collection » au-dessus du « rédacteur en chef » donne par ailleurs à penser qu'il est prévu de publier une édition de Word Routes adaptée aux locuteurs de beaucoup d'autres langues que le français.

De même, on se demande pourquoi alors que les titres des grandes catégories bénéficient de traduction, ceux des sous-catégories, souvent plus difficiles à saisir sont donnés uniquement en français. Ainsi le titre de la catégorie 156 Baked and Dried Foods, est traduit par Aliments cuits au four et Aliments secs, tandis que la souscatégorie 156.4 Féculents reste sans son équivalent anglais.

9 Au total, un tel ouvrage nous aidera certainement à faire comprendre aux étudiants que le vocabulaire ne s'apprend pas efficacement par liste et par cœur, mais qu'il se construit par champ, par réseau sémantique. Enfin, que de recherches fastidieuses vont être épargnées au lycéen curieux qui, jusqu'à présent, devait tourner de nombreuses pages pour découvrir les « four-letter words » les plus appropriés pour émailler sa prose - ici il les trouvera presque tous regroupés dans la catégorie 199 Sexe !

\section{BIBLIOGRAPHIE}

Summers D. (dir.). 1993. Longman Language Activator: The World's First Production Dictionary.

Londres : Longman.

\section{AUTEURS}

\section{LESLEY GRAHAM}

Université Bordeaux 2. lesley.graham@u-bordeaux2.fr 